\section{Maladies} génétiques

\title{
Les levures au service des maladies peroxysomales
}

Les maladies peroxysomales: groupes de complémentation

Quant aux maladies peroxysomales, elles sont divisées en trois groupes [2]. Le groupe A comprend des maladies sévères à début précoce comme la maladie de Zellweger $\left(\mathrm{m} / \mathrm{s} n^{\circ} 6\right.$, vol. 4, p. 391), l'adrénoleucodystrophie néonatale (NALD), la maladie de Refsum infantile (IRD), et l'acidémie hyperpipécolique. Biologiquement, elles se caractérisent, d'une part, par une accumulation d'acides phytanique, pristanique et pipécolique, ainsi que d'acides gras à très longue chaîne, et d'autre part par un déficit de synthèse des acides biliaires et des plasmalogènes, groupe d'éthers phospholipides à fonction antioxydante représentant plus de $20 \%$ des phospholipides chez l'homme [3-5]. A l'échelon cellulaire, de nombreuses enzymes peroxysomales sont absentes. Le groupe B n'est représenté que par la chondrodysplasie rhizomélique ponctuée (RCDP). On y retrouve l'accumulation d'acide phytanique, mais en revanche les acides gras à très longue chaîne ne sont pas présents dans le sérum. A l'échelon cellulaire, sont absentes, en une triade caractéristique, les deux enzymes catalysant les premiers stades de la synthèse du plasmalogène (la dihydroxy-acétone phosphate acyltransférase ou DHAP-AT et l'alkyl dihydroxy-acétone-phosphate synthase ou alkyl DHAP synthase) et la phytanique acide oxydase. Cliniquement la chondrodysplasie ponctuée se caractérise par un trouble de la croissance de type rhizomélique*, une cataracte, des calcifications épiphysaires

\footnotetext{
* Retard de croissance des régions proximales des
} membres. et une ichthyose** dans la forme classique; des formes mineures ont aussi été rapportées.

Le groupe C est représenté par l'adrénoleucodystrophie liée à l'X et par des maladies liées au déficit d'une seule enzyme peroxysomale (maladie de Refsum adulte, déficit en DHAT-AT ou dihydroxy-acétone phosphate acyltransférase, déficit d'une des enzymes de la $\beta$-oxydation peroxysomale).

Chez les malades des groupes A et B, qui décèdent généralement dans la première décennie, il fut possible, par l'établissement de lignées de lymphoblastes, de créer des groupes de complémentation. Ces groupes, de GG1 à CG4, et de CG6 à CG10, permettent de classer les malades du groupe A. Mais, dans un seul groupe de complémentation, on peut trouver différentes maladies, tandis que la plupart des malades atteints de chondrodysplasie rhizomélique ponctuée appartiennent au groupe de complémentation CG11.

\section{Séquences signal ciblant les peroxines à la membrane du peroxysome}

Au cours des années 1980, il fut démontré que les cellules de malades des groupes A et B n'étaient pas dépourvues de peroxysomes comme on le pensait initialement (en l'absence d'import de la catalase, les peroxysomes sont indétectables par les méthodes conventionnelles d'histochimie), mais que ceux-ci se présentaient comme des membranes vides (ghosts). L'hypothèse d'un trouble de l'acheminement des protéines du cytosol à la matrice peroxy-

\footnotetext{
** Retard de croissance des régions proximales des membres.
} appellation semblerait éminemment souhaitable. 
somale, par des voies différentes et multiples pour le groupe A, univoques pour le groupe $B$ semblait donc très probable [6]. Un début de confirmation fut apporté par la découverte fortuite d'une enzyme de la luciole, la luciférase. Cette protéine de la matrice peroxysomale se fixe par un signal cible comportant un tripeptide à l'extrémité carboxyterminale: SLK (Ser-Leu-Lys). De très nombreuses autres séquences de ce type furent retrouvées et regroupées sous le vocable de PTS1 (pour peroxisomial targeting signal). Un autre signal cible, PTS2, constitué d'une séquence située à l'extrémité aminoterminale $(\mathrm{R} / \mathrm{K})(\mathrm{L} / \mathrm{V}) \mathrm{X} 5 \mathrm{HL}$ ou [(Arg/Lys) (Leu/Val) X5-Hist-Leu] est présent sur la ketothiolase peroxysomale et la phytanate hydroxylase. Enfin, récemment, des PTS membranaires putatifs de protéines de membrane peroxysomale, ont été identifiés [7].

L'utilisation de la luciférase, protéine indicatrice hétérologue, pour explorer les séquences PTS1 et PTS2 fusionnées à la chloramphénicol acétyl transférase (enzyme CAT) permit de démontrer que chez les malades des groupes A et B, l'importation des protéines contenant PTS1, ou PTS2, ou les deux à la fois, était totalement ou partiellement défectueuse [8].

La conservation, au cours de l'évolution de ces signaux cibles peroxysomaux laissait espérer qu'il en serait de même pour les peroxines identifiées dans les levures ainsi que pour les gènes correspondants. De fait, sur les 17 PEX isolés jusqu'à présent, 7 orthologues humains furent trouvés, dont 5 sont impliqués dans des maladies peroxysomales (Tableau I). Parmi ceux-ci, les deux premiers furent identifiés par une équipe japonaise qui eut recours à la technique de complémentation: PEX2 codant pour une protéine de membrane liant le zinc qui complémente le CG10 [9], et PEX6, codant pour une ATPase putative qui complémente le groupe CG4 [10]. Puis l'homologue humain de $P E X 7$, d'abord découvert dans des levures, en particulier dans S. cerevisiae [11] qui est dépourvue de PTS2, se révéla être le gène codant pour le récepteur PTS1. Les mutations du gène $P E X 5$ se rencontrent chez des malades appartenant au groupe CG2 [12]. La protéine PEX 5 reconnaît les protéines contenant PTS1 présentes dans le cytosol et les achemine vers le peroxysome [13].

Il restait à trouver l'orthologue humain de la deuxième voie d'importation, codant pour PTS2, sachant que, dans les levures, c'est le mutant PEX7 qui affecte cette voie.

\section{$P E X 7$, le récepteur du signal}

Grâce au développement des banques de données pour les séquences exprimées, il est désormais plus facile de rechercher in silicio les orthologues humains des gènes isolés dans les levures. Il en fut ainsi pour le gène $P E X 7$ humain qui vient d'être découvert par deux équipes américaines $[13,14]$ et une équipe hollandaise [16] dont les travaux, publiés simultanément, fournissent des résultats convaincants.

Par recherche d'analogie de séquence avec des EST (expressed sequence tags), les gènes $P E X 7$ humain et murin furent identifiés. PEX7, localisé en 6q22-24, code pour une protéine de la sous-famille des protéines WD40* comprenant une trentaine de membres ayant des activités diverses (transmission des signaux, transport vésiculaire, contrôle du cycle cellulaire, assemblage du cytosquelette, régulation transcriptionnelle) mais dont aucun, jusqu'à présent, n'était impliqué dans la biogenèse peroxysomale. La protéine prédite comporte 323 acides aminés, avec une séquence N-terminale suivie de six motifs consécutifs WD40.

Comme on pouvait s'y attendre pour un gène d'un organite ubiquitaire, le transcrit est retrouvé dans tous les tissus étudiés. Mais, par Northern blot, on observe plusieurs autres transcrits plus petits pouvant correspondre à un épissage alternatif aboutissant, soit à des protéines porteuses d'une délétion les rendant non fonctionnelles, soit à des isoformes jouant un rôle régulateur dans des interactions entre diverses protéines.

\footnotetext{
* Protéines constituées de motifs très conservés comprenant une séquence d'environ 40 acides aminés se terminant par Trp-Asp (W-D), qui n'existent que chez les eucaryotes.
}

Après construction d'un vecteur d'expression codant pour pHsPEX (protéine PEX d'Homo sapiens) et transfection avec un plasmide exprimant PTS2 (obtenu par fusion de la séquence N-terminale PTS2 de la thiolase peroxysomale avec l'enzyme CAT), des études par immunofluorescence indirecte furent réalisées pour observer la distribution dans les cellules. Dans les fibroblastes normaux, à l'aide d'un anticorps antiCAT, on trouve une distribution caractéristique des peroxysomes. Dans des fibroblastes de malades atteints de chondrodysplasie rhizomélique ponctuée (RCDP), le couplage PTS2-CAT reste diffus dans le cytosol et PEX7 restaure l'aspect caractéristique de la distribution peroxysomale. Des mutations chez des malades atteints de RCDP furent observées dont l'une, une transversion $\mathrm{T} \rightarrow \mathrm{A}$, avec pour conséquence une mutation non-sens L292ter, est très fréquente (presque $50 \%$ des malades). Mutation récurrente ou effet fondateur? L'absence de cytosine méthylée, de séquences répétitives ou palindromiques, plaide plutôt en faveur de la deuxième proposition. Il est certes nécessaire d'étudier les haplotypes d'autres malades afin de mieux répertorier l'ensemble des mutations mais si l'existence d'un effet fondateur se confirmait, il pourrait se situer de préférence en Europe de Nord.

PEX7 est donc bien le récepteur peroxysomal PTS2 et ses mutations sont responsables de la chondrodysplasie rhizomélique ponctuée (à l'exception des formes qui résultent de déficiences isolées d'une des enzymes de la voie de synthèse des plasmalogènes : DHAP-AT ou alkykDHAP synthase, entre autres).

La localisation initiale de la protéine PEX7 reste encore controversée: cytosolique ou peroxysomale. Les derniers travaux la situeraient plutôt dans le cytosol où elle fixerait les protéines à transporter et où elle ferait la navette pour les amener à la matrice peroxysomale. PEX5 et PEX7 peuvent interagir puisque ces deux PEX appartiennent respectivement aux familles TPR (tetratricopeptide repeat) et WD40 connues pour leurs fréquentes interactions. En outre, un 
Tableau I

CLASSIFICATION DES GÈNES PEX

\begin{tabular}{|c|c|c|c|c|c|}
\hline Gène $P E X$ & Nature de la peroxine & Ancien nom & Maladie & CG & Localisation \\
\hline PEX1 & ATPase & ScPAS1 & Groupe A & CG1 & \\
\hline PEX2 & $\begin{array}{l}\text { Protéine de membrane } \\
\text { intégrale liant le } \mathrm{Zn}\end{array}$ & HsPAF1 & Groupe A & CG10 & $8 q 21.1$ \\
\hline PEX3 & $\begin{array}{l}\text { Protéine de membrane } \\
\text { sans similitude }\end{array}$ & ScPAS3 & & & \\
\hline PEX4 & $\begin{array}{l}\text { Enzyme conjugant } \\
\text { l'ubiquitine }\end{array}$ & ScPAS2 & & & \\
\hline PEX6 & $\begin{array}{l}\text { ATPase } \\
\text { cytoperoxydase et peroxydase }\end{array}$ & HsPXAAA1 & Groupe A & CG4 & $6 p 22-11$ \\
\hline PEX7 & $\begin{array}{l}\text { Récepteur PTS2 } \\
\text { répétition WD40 }\end{array}$ & ScPAS7 & $\begin{array}{l}\text { Groupe B } \\
\text { RCDP }\end{array}$ & CG11 & $6 q 22-24$ \\
\hline PEX8 & $\begin{array}{l}\text { Contient un signal } \\
\text { PTS } 12\end{array}$ & PpPER3 & & & \\
\hline PEX11 & $\begin{array}{l}\text { Protéine de prolifération } \\
\text { des peroxysomes }\end{array}$ & ScPMP27 & & & \\
\hline PEX12 & $\begin{array}{l}\text { Protéine de membrane } \\
\text { intégrale liant le } \mathrm{Zn}\end{array}$ & $\begin{array}{l}\text { PpPAS10 } \\
\text { Hs }\end{array}$ & Groupe A & CG3 & \\
\hline PEX13 & $\begin{array}{l}\text { Protéine de membrane } \\
\text { contenant SH3 } \\
\text { arrimage de PEX5p }\end{array}$ & $\begin{array}{l}\mathrm{Sc} \\
\mathrm{Hs}\end{array}$ & & & \\
\hline PEX14 & $\begin{array}{l}\text { Protéine de membrane } \\
\text { arrimage de PEX5p et de PEX7p }\end{array}$ & $\begin{array}{l}\mathrm{Sc} \\
\mathrm{Hs}\end{array}$ & & & \\
\hline
\end{tabular}

Peroxines recensées à ce jour, dont 8 retrouvées chez l'homme, 6 étant impliquées dans une maladie peroxysomale. (D'après[1] et [18].) CG: groupe de complémentation; Sc: Saccharomyces cerevisiae; Pp: Pichia pastoris; Hs: Homo sapiens; YI: Yarrowia lipolytica.

Le groupe A comprend les CG de 1 à 10 dans lesquels se trouvent le syndrome de Zellweger (ZS), I'adrénoleucodystrophie néonatale (NALD), la maladie de Refsum infantile (IRD) et l'acidémie hyperpipécolique.

Le groupe B comprend la chondrodystrophie rhizomélique ponctuée (RCDP) et correspond presque exclusivement au GC11.

cas de déficit peroxysomal en protéines à signal PST2 a été observé chez un malade où PEX5 n'était pas exprimé. Dans Saccharomyces cerevisiae, PEX5 n'intervient que pour l'importation des protéines à PTS1, mais elle a besoin d'une protéine d'arrimage, PEX 13 [17].

\section{Et maintenant PEX12}

Indépendamment de ces voies d'importation, et toujours par recherche in silicio, le gène $P E X 12$, orthologue de ScPEX12 vient d'être isolé chez l'homme. Il est impliqué dans le groupe de complémentation CG3, et des mutations furent trouvées chez deux malades non apparentés. Il s'agit, cette fois, d'une pro- peroxysomale qui restaure l'importation des protéines PTS1 et PTS2. Elle doit permettre le passage transmembranaire de ces protéines qui, en son absence, s'accumulent à la surface des peroxysomes [18].

De manière similaire, des mutations du gène $P E X 1$ viennent d'être identifiées parmi des patients appartenant au groupe GC1, groupe de complémentation qui comporte le plus grand nombre de malades du groupe A.

Sur les onze groupes de complémentation identifiés, un gène PEX responsable a déjà été trouvé pour six d'entre eux. L'approche in silicio devrait permettre de combler rapidement les manques. Il restera cependant à déterminer de manière précise comment les différentes peroxines s'assemblent pour former une machinerie d'import fonctionnelle. L'énorme travail de défrichage réalisé par les levuristes facilite grandement les recherches sur les peroxysomes humains et laisse espérer, pour un avenir proche, une totale compréhension des maladies peroxysomales dont l'abord a paru longtemps incertain

\section{Simone Gilgenkrantz}

2, rue Basse, 54330 Clerey-Brenon, France.

\section{TIRÉS À PART}

S. Gilgenkrantz. 
Note ajoutée aux épreuves

- Par invalidation de Pex5, un modèle murin pour le syndrome de Zellweger vient d'être obtenu qui sera très précieux pour une complète compréhension de la maladie humaine [20]. Les premiers résultats montrent qu'outre les protéines à PTS1, les protéines à PTS2 sont aussi touchées. Quant au retentissement sur le développement du cortex cérébral, il apparaît que le trouble de migration neuronale, cause des hétérotopies sous-corticales, s'accompagne d'une mort apoptotique des neurones, conséquence probable de leur défaut de migration et de différenciation.

- Deux nouvelles publications viennent de faire état de la découverte du gène de la maladie de Refsum [21, 22].

\section{RÉFÉRENCES}

1. Distel B, Erdmann R, Gould SJ, Blobel G, Crane DI, Cregg JM, Dodt G, et al. A unified nomenclature for peroxixome biogenesis factors. J Cell Biol 1996; 135: 1-3.

2. Wiemer EA, Subramani S. Protein import deficiencies in human peroxisomal disorders. Mol Genet Med 1994; 4: 119-52.

3. Lepage G, Kneepkens CMF, Roy CC. Dysfonction peroxysomale associée à la déficience en acides gras essentiels. Med Sci 1993; $9: 571-6$.

4. Boeymans J, Panneels V, van Sande J, Braekman J. Un rôle physiologique des plasmalogènes: la protection contre le stress oxydatif et l'excès d'iode. Med Sci 1995; 11: 254-9.

5. Caira F, Cherkaoui Malki M, Latruffe N. L'enzyme bifonctionnelle hydratase/déshydrogénase et l'oxydation peroxysomale des acides gras à très longue chaîne. Med $\mathrm{Sci}$ $1995 ; 11: 1131-9$.
6. Lazarow PB, Moser HW. Disorders of peroxisome biogenesis. In: Scriber CR, Beaudet AL, Sly WS, Valle D, eds. The metabolic basis of inherited diseases 6th ed. New York: McGraw Hill, 1989: 1479-509.

7. Wiemer EA, Luers GN, Faber KW, Wenzel T, Neenhuis M, Subramani S. Isolation and characterisation of Pex2p, a peroxisomal membrane protein essential for peroxisome biogenesis in the methylotrophic yeast Pichia pastoris. J Biol Chem 1996; 271 : $18973-$ 80.

8. Slawecki M, et al. Identification of three distinct peroxisomal protein import defects in patients with peroxisomal disorders. $J$ Cell Sci 1995; 108 : 1817-29.6.

9. Tsukamoto T, Muira S, Fujiki Y. Restoration by a $35 \mathrm{~K}$ membrane protein peroxisome assembly in a peroxisome deficient mammalian cell mutant Nature 1991; 350: 77-81.

10. Tsukamoto T, Muira S, Nakai T, Yokoto S, Shimozawa N, Susuki Y, Orii T, Fujiki Y, Sakai F, Bogaki A, et al. Peroxisome assembly factor-2, a putative ATPase cloned by functional complementation on a peroxisome-deficient mammalian cell mutant. Nat Genet 1995; 11 : 395-401.

11. van den Leij J, Franse MM, Elgersma Y, Distel B, Tabak HF. PAS10 is a tetracopeptide-repeat protein that is essential for the import of most matrix proteins into peroxisome of Saccharomyces cerevisiae. Proc Natl Acad Sci USA 1993; 90 : 11782-6.

12. Dogt G, Braverman N, Wong C, Moser A, Moser MW, Watkins P, valle D, Gould SJ. Mutations in the PTS1 receptor gene, DXR1, define complementation group 2 of the peroxisome biogenesis disorders. Nat Genet 1995; 9 : 115-24.

13. Rehling $\mathrm{P}$. The import receptor for the peroxisomal targeting signal 2 (PTS2) in Saccharomyces cerevisiae is encoded by the PAS7 gene. EMBO J 1996 ; 15 : 2901-13.
14. Purdue PE, Zhang JW, Skoneczny M, Lazarow PB. Rhizomelic chondrodysplasia punctata is caused by deficiency of human PEX7, a homologue of the yeast PTS2 receptor. Nat Genet 1997; 15 : 381-4.

15 Braverman N, Steel G, Obie C, Moser A, Moser H, Gould SJ, Valle D. Human PEX7 encodes the peroxisomal PTS2 receptor and is responsible for rhizomelic chondrodysplasia punctata. Nat Genet 1997; 15: 369-76.

16. Motley AM, Hettema EH, Hogenhout EM, Brites P, ten Asbroek LMA, Frits AW, et $a l$. Rhizomelic chondrodysplasia punctata is a peroxisomal protein targeting disease caused by non-functional PTS2 receptor. Nat Genet 1997 ; 15 : 377-80.

17. Egerlsma YL, Kwast L, Klein A, VoornBrouwer T, van den Berg M, Metzig B, America $\mathrm{T}$, Tabak HF, Distel $\mathrm{B}$. The $\mathrm{SH} 3$ domain of the Saccharomyces cerevisiae peroxisomal membrane protein Pex13p functions as a docking site for Pex5p, a mobile receptor for the import of PTS1 containing proteins. J Cell Biol 1996; 135 : 97-109.

18. Chang C-C, Lee WH, Moser H, Valle D, Gould SJ. Isolation of the PEX12 gene, mutated in group 3 of the peroxisome biogenesis disorders. Nat Genet 1997; 15 : 385-8.

19. Subramani S. PEX genes on the rise. Nat Genet 1997; 15: 331-2.

20. Baco M, Gressens P, Baumgzart E, Camerliet P, Casteels M, Fransen M, Evrard $\mathrm{P}$, et al. A mouse model for Zellweger syndrome. Nat Genet 1997; 17: 49-57.

21. Mihalik SJ, Morrell JC, Kim D, Sacksteder KA, Watkins PA, Gould SJ. Identification de PAHX, a Refsum disease gene. Nat Genet 1997; 17: 185-9.

22. Jansen GA, Ofman R, Ferdinandusse S, Ijlst L, Muijsers AO, Skekdal OH, et al. Refsum disease is caused by mutations in the phytanoyl-CoA hydroxylase gene. Nat Genet 1997: 17: 190-3.

\section{0 \\ Salon Franco-Israélien de Médecine et de Technologie Médicale 6 - 7 - 8 décembre 1997 - Maison France Israël - Paris Inauguration le 6 décembre 1997 à 21 h 30 Thème LE PROGRÈS Évolution et avancées des connaissances médicales Organisé par l'AMIF (participation des pharmaciens et dentistes) Pour renseignements et participation aux Tables rondes - tél. : 0149100910}

